\title{
Mutational status predicts the risk of thromboembolic events in lung adenocarcinoma
}

\author{
Elsa Davidsson ${ }^{1 \dagger}$, Nicola Murgia ${ }^{2 \dagger}$, Cristian Ortiz-Villalón ${ }^{3,4}$, Emil Wiklundh ${ }^{1,5}$, Magnus Sköld ${ }^{1,5}$, \\ Karl Gustav Kölbeck ${ }^{1,5}$ and Giovanni Ferrara ${ }^{1,5^{*}}$ (i)
}

\begin{abstract}
Background: Precision medicine promises to improve prognosis of patients affected by untreatable diseases. Patients with lung cancer (especially lung adenocarcinoma) bear an increased risk of VTE. Mutations in the EGFR and rearrangement in the ALK genes identify specific subgroups of patients. Aim of this study was to investigate the role of epidermal growth factor receptor (EGFR) and anaplastic lymphoma kinase (ALK) mutational status on the risk of venous thromboembolism (VTE) in lung adenocarcinoma.

Methods: A retrospective longitudinal design was used. Patients with lung adenocarcinoma diagnosed and undergoing a mutational analysis at the Karolinska University Hospital, Stockholm, Sweden between January 2009 and September 2015 were divided in three subgroups based on their mutational status (EGFR-, ALK-mutated, unexposed group). Event-free time for VTE was assessed using Cox regression analysis based on mutation status and treatment received.

Results: Three hundred-ten patients were included. A VTE occurred in 70 (22.6\%) patients. Mutation of EGFR was associated with a decreased risk of VTE (HR 0.46, 95\% Cl 0.23-0.94). Treatment with tyrosine kinase inhibitors (TKI) reduced the risk of VTE compared to other treatment strategies not including TKI (HR 0.42, 95\% Cl 0.29-0.79).

Conclusions: Our study suggests that patients with lung adenocarcinoma bearing a EGFR-mutation have a decreased risk of VTE compared with patients with other forms of lung adenocarcinoma. Targeted therapy with TKI alone or in combination with other treatments seems to reduce the risk of VTE compared to other treatments not including TKI.
\end{abstract}

Keywords: Non-small cell lung cancer, Thromboembolism, Mutation, Tyrosine kinase inhibitors, Precision medicine

\section{Background}

Precision medicine is rapidly changing the way chronic and untreatable diseases are managed [1]; it relies on the possibility to characterize patients at a molecular level and to offer them the best treatment at the right time based on the intrinsic mechanisms of their disease [2].

Lung cancer is the leading cause of cancer-related death for both men and women [3, 4]. In Sweden, the 5 -year survival is still $13.6 \%$ among men and $19.4 \%$ among women [4]. Lung adenocarcinoma is the most

\footnotetext{
* Correspondence: giovanni.ferrara@ki.se

${ }^{\dagger}$ Equal contributors

${ }^{1}$ Department of Respiratory Medicine and Allergy, Karolinska University Hospital, Stockholm, Sweden

${ }^{5}$ Department of Medicine Solna, Karolinska Institutet, Karolinska University Hospital, Stockholm 17176, Sweden

Full list of author information is available at the end of the article
}

common type of lung cancer in non-smokers, as well as in smokers and ex-smokers [2,3]. Molecular analyses to detect epidermal growth factor receptor (EGFR) mutations and anaplastic lymphoma kinase (ALK) rearrangement identify specific subgroups of patients $[5,6]$, often younger and non-smokers compared to other forms of adenocarcinoma [7-9]. Targeted treatments with tyrosine kinase inhibitors (TKI) have become the first line therapy for patients with advanced lung adenocarcinoma bearing EGFR mutations or ALK rearrangement, thanks to the improved clinical response and tolerability profile of these drugs compared to standard chemotherapy [10, 11]. By inhibiting the signaling of mutated receptor tyrosine kinases, TKI target the pathogenic mechanisms of the disease [12, 13]. Unfortunately, a main problem is the development of resistance to TKI during treatment [14]. 
The association between cancer and thromboembolism was first described in 1865 by Armand Trousseau, and the term Trousseau's syndrome is still used to describe a state of hypercoagulability in cancer patients [15]. Thromboembolic disease is a common cause of death among these patients $[15,16]$, and those with lung cancer are among the cancer groups with the highest risk of deep venous thrombosis (DVT) or venous thromboembolism (VTE) [17]. Lung adenocarcinoma is associated with a higher risk of VTE compared with other types of lung cancer [17-19]. It is still unknown how the EGFR and ALK mutational profile affects the risk of DVT/VTE in lung adenocarcinoma. The aim of this study was therefore to evaluate the occurrence of DVT/VTE in patients with EGFR- and ALK-mutated forms of lung adenocarcinoma compared with non-mutated cases. Furthermore, we evaluated the potential impact of different treatment strategies on the risk of VTE.

\section{Methods \\ Patients}

In the Stockholm County (approximately 2.2 million inhabitants), Sweden, diagnostic work-up and treatment of lung cancer is centralized at the Karolinska University Hospital in Stockholm. A list of all the mutational analysis tests for lung adenocarcinoma performed between January 1, 2009 and September 15, 2015 at the Department of Pathology of the Karolinska University Hospital was retrospectively reviewed in order to include all the patients bearing one of the EGFR mutations (EGFR group) or the ALK rearrangement (ALK group); the unexposed group included patients who tested negative for any mutation (EGFR, KRAS, ROS-1, BRAF mutations or ALK rearrangement) randomly extracted from the same list. Patients with other forms of lung cancer than adenocarcinoma were excluded.

Mutational status was tested for all the patients with Cobas $^{\oplus}$ EGFR Mutation test kit (CE-IVD) (Roche Molecular Systems, Inc., Branchburg, NJ, USA) for the EGFR mutations and with immunohistochemistry (Ventana Medical Systems, Inc, Tucson, AZ, USA) and fluorescence in situ hybridization (FISH) (Abbott Molecular, Des Plaines, IL, USA) for the ALK rearrangement.

\section{Data collection}

Data were retrospectively collected from the patients' electronic medical records with coded anonymous IDs. The following variables were collected for each patient: Age, gender, survival and co-morbidity (diabetes, hypertension); smoking status: Never-smoker (smoked less than 6 months), ex-smoker (have not smoked in the last 6 months) and current smoker; tumor-related variables: Stage according to TNM classification, mutational status and main treatments; target events: VTE or DVT, time from diagnosis, on-going anti-coagulant treatment (preventive dosage) and time from death; performance status 0-4 (assessed according to World Health Organization/ Eastern Cooperative Oncology Group) [20]. Target events were all radiologically confirmed with computed tomography $(\mathrm{CT})$ with intravenous contrast (VTE) or ultrasound (DVT).

\section{Statistical analysis}

Descriptive analyses include mean and standard deviation of the mean (SD) for the continuous variables and proportions of the total for the categorical variables. Subgroup analyses were performed for each of the three study groups. For comparison of categorical variables $x^{2}$-test was used. Incidence rates and their confidence intervals were calculated for all the categorical variables, grouping treatment options in four categories (TKI alone, conventional chemotherapy alone, other treatments in combination with TKI, other treatments without TKI).

Event-free time for target events was assessed using Cox regression analysis according to mutation status and treatment, adjusting for other relevant factors (age, gender, smoking status, treatment with a preventive dose of low molecular weight heparin and stage). The choice of the covariates to adjust for was based on statistical relevance by Kaplan-Meier curves for the single covariate (e.g., age and gender) or by a biological relevance (e.g., anti-coagulant treatment). Two different Cox regression models, one with mutational status and one considering treatment options, were performed to avoid the expected excess of collinearity between these two variables. In these models, age was considered as a categorical variable with cut-off at 66 years as median age of the population. Patients with a target event at the time of diagnosis were excluded from the Cox analysis. Level of significance was set at $p<0.05$. Statistics were performed with SPSS 23 edition (IBM Corporation, Chicago, Illinois).

\section{Ethics}

The main investigator (GF) contacted the Regional Ethical Committee in the Stockholm County to discuss this study. The Committee considered this study as a quality control and quality improving process. As such, no ethical permission is necessary according to the Swedish law. The study was approved by the direction of the Department of Respiratory Medicine and Allergy, Karolinska University Hospital, Stockholm, Sweden, as a quality control and quality improving process at the Department.

\section{Results}

One thousand thirteen patients with lung adenocarcinoma underwent a mutational analysis test during the 
study period at the Karolinska University Hospital; among them, 104 (10.3\%) tested positive for a EGFR mutation, 52 (5.1\%) had an ALK translocation. The unexposed group included 154 (15.2\%) patients with a negative test result for any mutation. Demographics and clinical features of the enrolled population are showed in Table 1. In the entire study population, 159 (51.3\%) patients were in stage IV at diagnosis; more patients were diagnosed in stage IV in the mutated groups compared to the unexposed group $(p>0.001$, Table 1$)$. Performance status 0 was assessed in $203(65.5 \%)$ patients at diagnosis, meaning that most of the patients were fully active at that time; no statistical difference was observed among the groups (Table 1). As expected, there was a difference in smoking habits: both of the mutated groups had a higher number of never-smokers $(n=45,43.3 \%$ and $n=26,50 \%$ respectively in the EGFR- and ALKgroup compared to $n=15,9.7 \%$ in the unexposed group, $p<0.001)$ and the unexposed group had more current smokers $(n=52,33.8 \%$ compared to $n=9,8.7 \%$ and $n=6,11.5 \%$ in the EGFR- and ALK-group, respectively, $p<0.001)$.

In the whole study population, a target event occurred in 70 (22.6\%) patients: the course of the disease was complicated in $17(5.4 \%)$ patients by a DVT, in 51 by a VTE (16.5\%) and in two (0.7\%) patients by both DVT and VTE.

Eight $(7.7 \%)$ patients in the EGFR-group, four $(7.7 \%)$ in the ALK-group and five (3.2\%) in the unexposed group had a target event already at diagnosis. These 17 patients were therefore excluded, leaving 293 patients (94.5\% of the total study population) and 53 target events ( $75.7 \%$ of all the events) for the Cox regression analysis. The follow up time ranged between 10 and 3160 person/days. The sum of person/days in the whole population was 232,060 . Overall incidence rate was $0.23 \times 1000$ person/days $(95 \%$ CI $0.17-0.29)$. Frequencies of target events in relation to patients' demographics and characteristics at diagnosis are presented in Table 2.

Forty-three patients (14.7\% out of the 293 patients included in the Cox analysis, 37 patients in the EGFRgroup, four in the ALK-group and two in the unexposed group) received treatment with TKI only. Totally 58 (19.8\% of 293) patients, including two patients not bearing a EGFR- or ALK-mutation, received TKI as first line treatment. Fifty-six (38.8\%) and $40(27.8 \%)$ out of the 144 patients with confirmed EGFR- or ALK-mutation were treated with TKI as first or second line treatment, respectively.

Table 1 Demographics and clinical features of patients at time of diagnosis

\begin{tabular}{|c|c|c|c|c|c|}
\hline Variable & $\operatorname{EGFR}(n=104)$ & $\operatorname{ALK}(n=52)$ & $\begin{array}{l}\text { Unexposed group } \\
(n=154)\end{array}$ & Total $(n=310)$ & $p$-value \\
\hline Age mean $\pm S D$ & $66.9 \pm 12.0$ & $57.6 \pm 11.9$ & $67.4 \pm 9.4$ & $65.6 \pm 11.3$ & \\
\hline Gender: & & & & & NS \\
\hline Female & $68(65.4 \%)$ & $32(61.5 \%)$ & $86(55.8 \%)$ & $186(60.0 \%)$ & \\
\hline Male & $36(34.6 \%)$ & $20(38.5 \%)$ & $68(44.2 \%)$ & $124(40.0 \%)$ & \\
\hline Stage: & & & & & $<0.00$ \\
\hline । & $18(17.3 \%)$ & $4(7.7 \%)$ & $41(26.6 \%)$ & $63(20.3 \%)$ & 1 \\
\hline$\|$ & $8(7.7 \%)$ & $1(1.9 \%)$ & $24(15.6 \%)$ & $33(10.6 \%)$ & \\
\hline III & 7 (6.7\%) & $13(25.0 \%)$ & 35 (22.7\%) & $55(17.7 \%)$ & \\
\hline IV & 71 (68.3\%) & 34 (65.4\%) & $54(35.1 \%)$ & $159(51.3 \%)$ & \\
\hline Performance status: & & & & & NS \\
\hline 0 & $67(64.4 \%)$ & 37 (71.1\%) & 99 (64.3\%) & $203(65.5 \%)$ & \\
\hline 1 & $33(31.7 \%)$ & $12(23.1 \%)$ & $46(29.9 \%)$ & $91(29.4 \%)$ & \\
\hline 2 & $2(1.9 \%)$ & $2(3.8 \%)$ & $5(3.2 \%)$ & 9 (2.9\%) & \\
\hline 3 & $2(1.9 \%)$ & $1(1.9 \%)$ & $4(2.6 \%)$ & $7(2.3 \%)$ & \\
\hline Smoking status: & & & & & $<0.00$ \\
\hline Never-smoker & 45 (43.3\%) & $26(50.0 \%)$ & $15(9.7 \%)$ & $86(27.7 \%)$ & 1 \\
\hline Ex-smoker & $50(48.1 \%)$ & $20(38.5 \%)$ & $87(56.5 \%)$ & $157(50.6 \%)$ & \\
\hline Smoker & $9(8.7 \%)$ & $6(11.5 \%)$ & $52(33.8 \%)$ & $67(21.6 \%)$ & \\
\hline Diabetes & $7(6.7 \%)$ & $2(3.8 \%)$ & $15(9.7 \%)$ & $24(7.7 \%)$ & NS \\
\hline Hypertension & $31(29.8 \%)$ & $11(21.2 \%)$ & $64(41.6 \%)$ & $106(34.2 \%)$ & 0.014 \\
\hline $\mathrm{AC}$ before the event & 7 (6.7\%) & 9 (17.3\%) & $19(12.3 \%)$ & 35 (11.3\%) & NS \\
\hline
\end{tabular}

Abbreviations: EGFR epidermal growth factor receptor, $A L K$ anaplastic lymphoma kinase, $S D$ standard deviation, $A C$ anticoagulants, preventive dose for venous thromboembolism 
Table $\mathbf{2}$ Incidence rate of target events (venous thromboembolism, deep venous thrombosis)

\begin{tabular}{|c|c|c|}
\hline Variable & Target event (n) & Incidence rate (95\% Cl) \\
\hline \multicolumn{3}{|l|}{ Mutation status: } \\
\hline EGFR+ & 15 & $0.21(0.10-0.32)$ \\
\hline ALK+ & 13 & $0.27(0.13-0.42)$ \\
\hline Unexposed group & 25 & $0.22(013-0.31)$ \\
\hline \multicolumn{3}{|l|}{ Gender: } \\
\hline Female & 31 & $0.21(0.14-0.29)$ \\
\hline Male & 22 & $0.26(0.15-0.37)$ \\
\hline \multicolumn{3}{|l|}{ Age } \\
\hline$<66$ years & 38 & $0.33(0.22-0.43)$ \\
\hline$>66$ years & 15 & $0.13(0.06-0.19)$ \\
\hline \multicolumn{3}{|l|}{ Stadium } \\
\hline I & 4 & $0.05(0.01-0.10)$ \\
\hline$\|$ & 6 & $0.21(0.04-0.37)$ \\
\hline III & 9 & $0.18(0.06-0.30)$ \\
\hline IV & 34 & $0.46(0.30-0.61)$ \\
\hline \multicolumn{3}{|l|}{ Smoking status: } \\
\hline Never smoker & 17 & $0.26(0.14-0.38)$ \\
\hline Ex-smoker & 14 & $0.18(0.11-0.26)$ \\
\hline Smoker & 22 & $0.26(0.14-0.38)$ \\
\hline Hypertension & 12 & $0.14(0.06-0.22)$ \\
\hline Diabetes & 0 & - \\
\hline$A C$ before the event & 7 & $0.24(0.06-0.42)$ \\
\hline \multicolumn{3}{|l|}{ Treatment options: } \\
\hline CTP alone & 30 & $1.00(0.55-1.45)$ \\
\hline Other treatment without TKI & 4 & $0.11(0.05-0.17)$ \\
\hline Other treatment with TKI & & $0.25(0.13-0.38)$ \\
\hline TKI alone & 19 & $0.15(0.04-0.29)$ \\
\hline
\end{tabular}

Incidence rate (x1000 person/days) in relation to mutation status, baseline clinical features, and treatment options

Abbreviations: EGFR+ patients bearing a mutation in the epidermal growth factor receptor (EGFR), $A L K+$ patients bearing a rearrangement of the anaplastic lymphoma kinase (ALK), AC anticoagulants, preventive dose for venous thromboembolism, CTP standard chemotherapy, TKI tyrosine kinase inhibitors
Forty-two patients (14.3\% out of the 293 patients included in the Cox analysis, five patients in the EGFR group, eight in the ALK group and 29 in the unexposed groups) received treatment with chemotherapy only. Table 3 shows data about treatment-combinations in the study population divided according mutational status.

The Cox regression analysis identified variables affecting the risk of DVT/VTE: Mutational status was associated with a lower risk of target events for the EGFR group, as well as age $>66$, while stage IV was associated with an increased risk (Table 4). Adjusting also for smoking status did not alter the results (data not shown).

Weighed Cox regression analysis was used to calculate the event-free time for DVT/VTE for the three groups according to mutational status. The unexposed group had a significantly shorter time to first event compared to mutated groups (Fig. 1).

Another Cox regression analysis considering the role of treatments confirmed the protective role of age $>66$ and the increased risk of target events in patients with disease in stage IV; all other treatment strategies compared with the reference group chemotherapy alone seemed to exert a protective effect on the risk of target events (Table 5).

A sensitivity analysis was performed considering only patients with disease in stage IV: treatment with TKI alone (HR 0.12 95\% CI: 0.04-0.42) or in association with other treatments (HR $0.17 \quad 95 \%$ CI: 0.07-0.43) clearly resulted in a protective effect for target events when compared to chemotherapy alone, whilst no protective effect was noted for other approaches including chemotherapy. Age $>66$ was still protective (HR 0.38 95\% CI: $0.18-0.83$ ). Within this group of patients in stage IV, patients receiving chemotherapy alone or in combination with treatments other than TKI had a significantly shorter time to first event compared to patients treated with TKI during the clinical course of their disease (Fig. 2).

Table 3 Frequency of prescribed treatment and treatment combinations in the study population, grouped by mutational status

\begin{tabular}{lllll}
\hline Treatment & EGFR $(n=96)$ & ALK $(n=48)$ & Unexposed group $(n=149)$ & Total $(n=293)$ \\
\hline TKI & $37(38.5 \%)$ & $4(8.3 \%)$ & $2(1.3 \%)$ & $43(14.7 \%)$ \\
TKI + surgery & $4(4.2 \%)$ & $1(2.1 \%)$ & $0(0 \%)$ & $5(1.7 \%)$ \\
CTP & $5(5.2 \%)$ & $8(16.7 \%)$ & $29(19.5 \%)$ & $42(14.3 \%)$ \\
CTP + surgery & $0(0 \%)$ & $2(4.2 \%)$ & $34(22.8 \%)$ & $36(12.3 \%)$ \\
CTP + TKI & $26(27.1 \%)$ & $24(50 \%)$ & $0(0 \%)$ & $50(17 \%)$ \\
RTP & $2(2.1 \%)$ & $1(2.1 \%)$ & $13(8.7 \%)$ & $16(5.5 \%)$ \\
Surgery & $10(10.4 \%)$ & $3(6.3 \%)$ & $30(20.1 \%)$ & $43(14.7 \%)$ \\
Combination (TKI, CTP, RTP & $7(7.3 \%)$ & $3(6.3 \%)$ & $30(20.1 \%)$ & $40(13.7 \%)$ \\
and/or surgery) & & & $11(7.4 \%)$ & $18(6.1 \%)$ \\
No treatment & $5(5.2 \%)$ & $2(4.2 \%)$ & \\
\hline
\end{tabular}

Abbreviations: EGFR epidermal growth factor receptor, ALK anaplastic lymphoma kinase, TKI tyrosine kinase inhibitors, CTP chemotherapy, RTP radiotherapy 
Table 4 Cox regression analysis for risk of thromboembolic disease in lung adenocarcinoma

\begin{tabular}{ll}
\hline Variable & HR $(95 \% \mathrm{Cl})$ \\
\hline Gender & 1 \\
Male & $0.83(0.47-1.45)$ \\
Female & \\
Age & 1 \\
$\quad<66$ & $0.44(0.23-0.82)$ \\
$>66$ & \\
Stadium & 1 \\
I & $3.10(0.86-11.25)$ \\
॥ & $3.11(0.94-10.32)$ \\
III & $8.70(2.90-26.17)$ \\
IV & \\
AC before the target event & 1 \\
NO & $1,10(0.49-2.50)$ \\
YES & \\
Mutational status & 1 \\
Non exposed & $0.46(0.23-0.94)$ \\
EGFR+ & $0.61(0.29-1.29)$ \\
ALK+ &
\end{tabular}

Significant covariates are shown. Adjusted for gender $H R$ hazard ratio, 95\% Cl 95\% confidence interval, NS not significant, $A C$ anticoagulants, preventive dose for venous thromboembolism, EGFR+ patients bearing a mutation in the epidermal growth factor receptor (EGFR), ALK+ patients bearing a rearrangement of the anaplastic lymphoma kinase (ALK)

\section{Discussion}

The purpose of this study was to investigate if mutational status affected the risk of thromboembolism in patients with lung adenocarcinoma. The main outcome was that patients bearing a EGFR mutation had a lower risk of target events. This result was not confirmed for patients bearing a ALK rearrangement, but this was mainly due to the limited sample size of this group of patients ( $a$ trend for protection was observed in the main analysis reported in Table 4).

Treatment with TKI also appeared to further decrease the risk of thromboembolic events, both when used as the only treatment or in combination/sequence with other treatments during the course of the disease. Most mutated patients received TKI, which makes it difficult to fully distinguish the protective effect of the treatment itself.

Mutational status is independent of stage, whilst treatment with TKI is related to stage; this is why the protective effect of mutation is a more consistent finding. The result regarding TKI treatment is partially related to mutational status but is also due to the complexity of clinical management of lung cancer patients. Our study included patients who received both TKI and chemotherapy in different phases of the disease, as well as patients treated with only chemotherapy or chemotherapy in combination with treatments other than TKI. A potential limit is represented by the fact that the exact duration of TKI treatment or of other forms of treatment was not assessed in this study. Nevertheless, the unexposed group not only

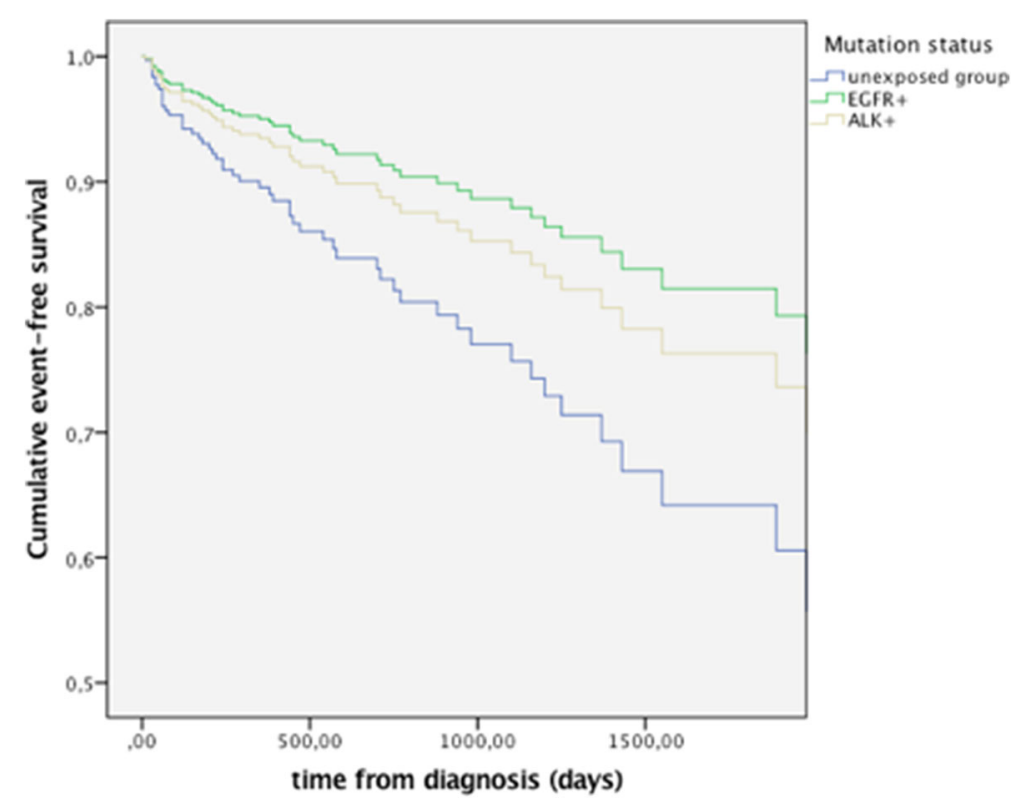

Fig. 1 Event-free survival of patients in the Epidermal Growth Factor Receptor (EGFR), anaplastic lymphoma kinase (ALK) mutated groups and in the unexposed group. Calculated with the same Cox regression model as used in Table 3. Demonstrates the difference in event-free survival between the unexposed and mutated groups. Cumulative event-free survival variable: time before the first thromboembolic event 
Table 5 Weighted cox regression analysis for variables affecting event-free time

\begin{tabular}{ll}
\hline Variable & HR $(95 \% \mathrm{Cl})$ \\
\hline Gender & 1 \\
Male & $0.88(0.50-1.55)$ \\
Female & \\
Age & 1 \\
$<66$ & $0.37(0.20-0.70)$ \\
$>66$ & \\
Stadium & 1 \\
I & $2.64(0.73-9.57)$ \\
II & $2.40(0.70-8.24)$ \\
III & $3.63(1.05-12.50)$ \\
IV & \\
AC before the target event & 1 \\
NO & $1,03(0.45-2.33)$ \\
YES & \\
Treatment options & 1 \\
CTP alone & $0.20(0.08-0.51)$ \\
Other treatment without TKI & $0.25(0.12-0.51)$ \\
Other treatment with TKI & $0.14(0.05-0.43)$ \\
TKI alone & \\
\hline HR & \\
\hline
\end{tabular}

$H R$ hazard ratio, $95 \% \mathrm{Cl} 95 \%$ confidence interval, $N S$ not significant, $A C$ anticoagulants, preventive dose for venous thromboembolism, CTP chemotherapy, TKI tyrosine kinase inhibitors had an increased risk of target events, but also the time to the first event event was shorter.

According to a preclinical study [21] on colorectal cancer cells, inhibition of EGFR decreases the expression of tissue factor; this would offer a stimulating hypothesis to explain our results; studies investigating specifically which tissue factors are affected in vivo by different treatments might result in the discovery and validation of biomarkers for treatment response and prediction of comorbidities like DVT/VTE.

A previous study by Lee et al. [22] investigated risk factors of thromboembolism in non-small cell lung cancer patients. No significant differences were found between mutated and non-mutated patients. Their study had several flaws for this purpose, e.g., not all patients were tested for mutations and the histology was not specifically adenocarcinoma. They did find an increased risk associated with TKI treatment and hypothesized that this could partly be explained by extensive chemotherapy treatment and longer survival in EGFR-mutated patients. Another study by Yang et al. [23] showed an increased DVT/VTE risk with TKI treatment. The aim of this study was to evaluate the effect of postoperative treatment on DVT/VTE on all types of lung cancer, and neither clinical stage nor other treatments were taken into account when analyzing the increased risk of target events with TKI. Those who received TKI in this study were in a more advanced stage and received extensive

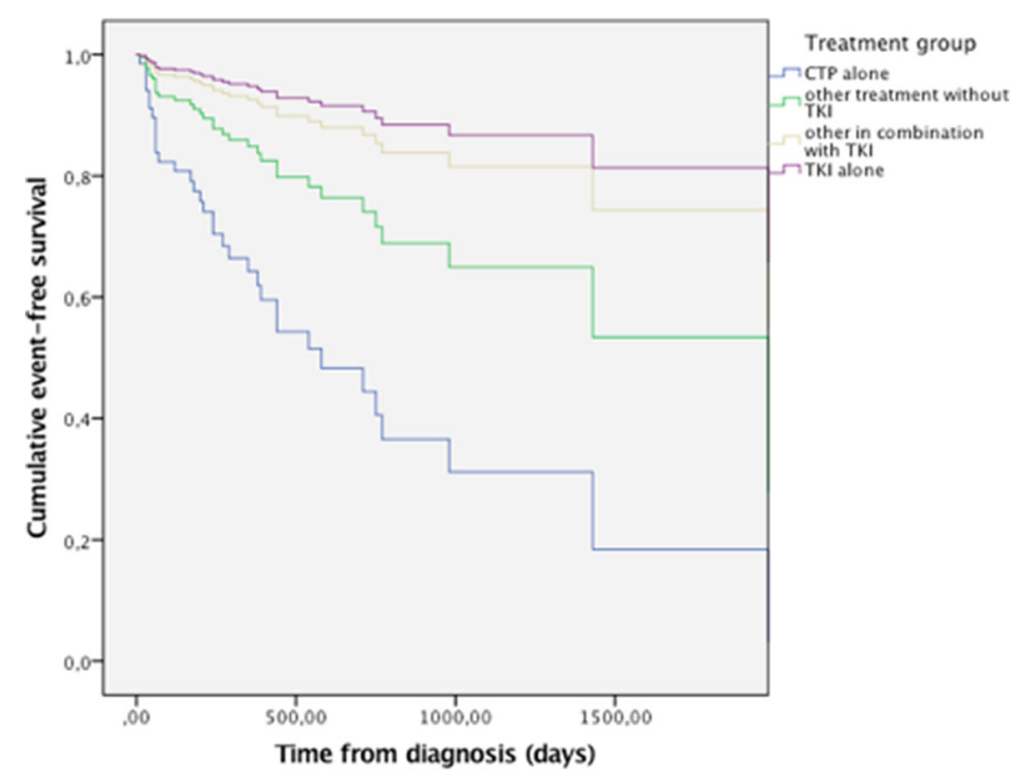

Fig. 2 Sensitivity analysis considering risk of thromboembolism for treatments received by patients with lung adenocarcinoma in stage IV during the study period. Calculated with a Cox regression model including only patients with lung adenocarcinoma in stage IV and considering chemotherapy alone as reference for the analysis. Demonstrates the difference in event-free survival between patients treated with tyrosine kinase inhibitors (TKI) alone or in combination with other treatments compared to other treatments not including TKI. CPT: chemotherapy; TKI: tyrosine kinase inhibitors. Cumulative event-free survival variable: time before the first thromboembolic event 
treatment, which likely affected the results. In addition, only 32 out of 1001 patients received TKI.

The finding that chemotherapy could increase the risk of DVT/VTE is in accordance with previous studies $[15,18,22,24]$, as well as the risk of DVT/VTE associated with advanced clinical stage $[15,24]$. The higher occurrence of thromboembolic events in our study compared to others [17-19] could depend on the fact that data were analyzed from a highly selected group of patients affected by lung adenocarcinoma. This group is known to have higher risk of DVT/VTE compared to other histotypes [17-19, 22]. Furthermore, treatment with cisplatin-based regimens is a strong risk factor for DVT/ VTE in patients with non-small cell lung cancer [25]; the vast majority of the patients included in this study were treated with cisplatin-based regimens, as these treatments are still the first choice in patients with advanced adenocarcinoma not bearing a EGFR- or ALK-mutation according to the current Swedish guidelines [11]. Another possibility is that mutated patients are more often diagnosed at an advanced stage; the follow up period was also longer compared to previous studies.

This was a retrospective study in which selection of patients was based on a list of mutational analyses obtained from the Pathology Department of the Karolinska University Hospital. This can raise the concern about a potential selection bias, as we did not enroll prospectively all the patients with lung adenocarcinoma diagnosed in the catchment area. Furthermore, we cannot exclude that not all the patients diagnosed with lung adenocarcinoma during the study period underwent molecular testing. Nevertheless, the proportion of EGFR and ALK mutations in the cohort used to select our study population is consistent with data reported in other series [26], and this should reinforce the validity of our findings.

A major strength of this study compared with previous studies [22, 23] on the risk of thromboembolism in patients with lung cancer is that it did include only patients with lung adenocarcinoma and that patients were specifically selected on the base of the results of the mutational analysis. In particular, the patients included in the unexposed group were truly negative at the molecular testing, confirming that they in fact did not bear any mutation. Previous studies $[22,23]$ have not found such a correlation but they included non-selected/non-tested populations.

In our study, older patients (with age $>66$ years) showed a decreased risk of thromboembolism. One reason could be that younger patients are treated more aggressively, with higher doses of chemotherapy. Another plausible explanation could be that the cancer itself is more aggressive in younger patients. The fact that age $>66$ is protective for DVT/VTE seems to contradict general risk factors for thromboembolism [15, 27], but previous studies support our finding $[17,19]$. This study did not get any significant results on association between performance status and VTE/DVT and previous studies have been inconclusive $[28,29]$.

A major limitation of this study was the sample size, especially for the ALK group. Another limitation of our design is that variables such as performance status, smoking status and clinical stage have been treated as if they were constant from diagnosis, when they can vary over time; furthermore, the study was designed to evaluate the risk of DVT/VTE in relation to mutation status and not specifically in association with treatment and variation of treatment over time; nevertheless, our results show that the treatment prescribed greatly influence the risk of target events, and this should be taken in consideration when managing the patients with higher risk.

\section{Conclusions}

Our study shows for the first time that there is a strong correlation between the risk of severe clinical complications and mutational status in lung adenocarcinoma, and that targeted therapies have a safer profile than systemic chemotherapy even for events not directly connected to cancer growth as thromboembolism; this study reinforces the concept that precision medicine is preferable in lung cancer [2]. Furthermore, international guidelines have so far failed to provide strong recommendation for the use of anticoagulants as prophylaxis of DVT/VTE in lung cancer (16); the results of our study can help to identify which patients with lung adenocarcinoma would benefit the most from such interventions.

\section{Abbreviations \\ 95\% Cl: 95\% confidence interval; ALK: Anaplastic lymphoma kinase; ATP: Adenosine triphosphate; CTP: Chemotherapy; DVT: Deep venous thrombosis; EGFR: Epidermal growth factor receptor; FISH: Fluorescence in situ hybridization; HR: Hazard ratio; RTP: Radiotherapy; SD: Standard deviation of the mean; TKI: Tyrosine kinase inhibitors; TNM: TNM classification of malignant tumours; VTE: Venous thromboembolism}

\section{Acknowledgements}

Not applicable.

Funding

Not applicable.

Availability of data and materials

The datasets used and/or analysed during the current study are available from the corresponding author on reasonable request.

Authors' contributions

$E D, N M$ and GF contributed to the study design, to the data collection, statistical analysis, interpretation of the data and drafting of the manuscript. COV, EW, KK, MS contributed substantially to the study design, data analysis and interpretation, and to the writing of the manuscript. All authors read and approved the final manuscript.

Competing interests

The authors declare that they have no competing interests.

Consent for publication

Not applicable. 


\section{Ethics approval and consent to participate}

Not applicable. Please read also the part on ethics in the methods.

\section{Publisher's Note}

Springer Nature remains neutral with regard to jurisdictional claims in published maps and institutional affiliations.

\section{Author details}

'Department of Respiratory Medicine and Allergy, Karolinska University Hospital, Stockholm, Sweden. ${ }^{2}$ Section of Occupational Medicine, Respiratory Diseases and Toxicology, University of Perugia, Perugia, Italy. ${ }^{3}$ Department of Pathology, Karolinska University Hospital, Stockholm, Sweden.

${ }^{4}$ Oncology-Pathology Unit, Karolinska Institutet, Stockholm, Sweden.

${ }^{5}$ Department of Medicine Solna, Karolinska Institutet, Karolinska University Hospital, Stockholm 17176, Sweden.

\section{Received: 21 February 2017 Accepted: 1 May 2017}

Published online: 03 July 2017

\section{References}

1. Mirnezami R, Nicholson J, Darzi A. Preparing for precision medicine. N Engl J Med. 2012;366:489-91.

2. Jameson JL, Longo DL. Precision medicine - personalized, problematic and promising. N Engl J Med. 2015;372:2229-34.

3. World Health Organization. Fact sheet N²97 [Internet]. [cited 2016 Dec 11]. Available from: http://www.who.int/mediacentre/factsheets/fs297/en/.

4. National Board of Health and Welfare. Cancer i siffror 2013. 2013; [cited 2016 Dec 11]. Available from: http://www.socialstyrelsen.se/ publikationer2013/2013-6-5.

5. Herbst RS, Heymach JV, Lippman SM. Lung cancer. N Engl J Med. 2008;359: 1367-80.

6. Travis WD, Brambilla E, Riely GJ. New pathologic classification of lung cancer: relevance for clinical practice and clinical trials. J Clin Oncol. 2013;31:992-1001.

7. Sonobe M, Manabe T, Wada H, Tanaka F. Mutations in the epidermal growth factor receptor gene are linked to smoking-independent, lung adenocarcinoma. Br J Cancer. 2005;93:355-63.

8. Kang HJ, Lim HJ, Park JS, Cho YJ, Yoon HI, Chung JH, et al. Comparison of clinical characteristics between patients with ALK-positive and EGFR-positive lung adenocarcinoma. Respir Med. 2014;108(2):388-94.

9. Kwak EL, Bang YJ, Camidge DR, Shaw AT, Solomon B, Maki RG, et al. Anaplastic lymphoma kinase inhibition in Non-small-cell lung cancer. N Engl J Med. 2010;363:1693-703.

10. Masters GA, Temin S, Azzoli CG, Giaccone G, Baker Jr S, Brahmer JR, et al. Systemic therapy for stage IV Non-small-cell lung cancer: american society of clinical oncology clinical practice guideline update. J Clin Oncol. 2015;33: 3488-515.

11. Regionala cancercentrum i samverkan. Nationellt vårdprogram Lungcancer [cited 2016 Dec 11]. Available from: https://www.cancercentrum.se/ globalassets/cancerdiagnoser/lunga-och-lungsack/vardprogram/natvp_ lungcancer_2015-03-10.pdf.

12. Gazdar AF. Personalized medicine and inhibition of EGFR signaling in lung cancer. N Engl J Med. 2009;361:1018-20.

13. Pall G. The next-generation ALK inhibitors. Curr Opin Oncol. 2015;27:118-24.

14. Rosell R, Karachaliou N. Lung cancer in 2014: Optimizing lung cancer treatment approaches. Nat Rev Clin Oncol. 2015;12:75-6.

15. Ikushima S, Ono R, Fukuda K, Sakayori M, Awano N, Kondo K. Trousseau's syndrome: cancer-associated thrombosis. Jpn J Clin Oncol. 2016;55:2049-53.

16. Farge D, Debourdeau P, Beckers M, Baglin C, Bauersachs RM, Brenner B, et al. International clinical practice guidelines for the treatment and prophylaxis of venous thromboembolism in patients with cancer. J Thromb Haemost. 2013;11:56-70

17. Chew HK, Davies AM, Wun T, Harvey D, Zhou H, White RH. The incidence of venous thromboembolism among patients with primary lung cancer. J Thromb Haemost. 2008;6:601-8.

18. Alexander M, Kirsa S, Wolfe R, MacManus M, Ball D, Solomon B, et al. Thromboembolism in lung cancer - an area of urgent unmet need. Lung Cancer. 2014:84:275-80.

19. Zhang Y, Yang Y, Chen W, Guo L, Liang L, Zhai Z, et al, China Venous Thromboembolism (VTE) Study Group. Prevalence and associations of VTE in patients with newly diagnosed lung cancer. Chest. 2014;146:650-8.
20. Young J, Badgery-Parker T, Dobbins T, Jorgensen M, Gibbs P, Faragher I, et al. Comparison of ECOG/WHO performance status and ASA score as measure of functional status. J Pain Symptom Manage. 2015;49:258-64.

21. Yu JL, May L, Lhotak V, Shahrzad S, Shirasawa S, Weitz JI, Coomber BL, Mackman N, Rak JW, et al. Oncogenic events regulate tissue factor expression in colorectal cancer cells: implications for tumor progression and angiogenesis. Blood. 2005;105:1734-41.

22. Lee YG, Kim I, Lee E, Bang SM, Kang CH, Kim YT, et al. Risk factors and prognostic impact of venous thromboembolism in Asian patients with non-small cell lung cancer. Thromb Haemost. 2014;111:1112-20.

23. Yang Y, Zhou Z, Niu XM, Li ZM, Chen ZW, Jian H, et al. Clinical analysis of postoperative venous thromboembolism risk factors in lung cancer patients. J Surg Oncol. 2012;106:736-41.

24. Corrales-Rodriguez L, Blais N. Lung cancer associated venous thromboembolic disease: a comprehensive review. Lung cancer (Amsterdam, Netherlands). 2012;75:1-8

25. Lee YG, Lee E, Kim I, Lee KW, Kim TM, Lee SH, et al. Cisplatin-based chemotherapy is a strong risk factor for thromboembolic events in small-cell lung cancer. Cancer Res Treat. 2015;47(4):670-5.

26. Korpanty GJ, Graham DM, Vincent MD, Leighl NB. Biomarkers that currently affect clinical practice in lung cancer: EGFR, ALK, MET, ROS-1, and KRAS. Front Oncol. 2014:4:204.

27. Lin J, Wakefield TW, Henke PK. Risk factors associated with venous thromboembolic events in patients with malignancy. Blood Coagul Fibrinolysis. 2006;17:265-70.

28. Agnelli G, Verso M, Mandala M, Gallus S, Cimminiello C, Apolone G, et al. A prospective study on survival in cancer patients with and without venous thromboembolism. Intern Emerg Med. 2014;9:559-67.

29. Wang Z, Yan HH, Yang JJ, Wang BC, Chen HJ, Zhou Q, et al. Venous thromboembolism risk factors in Chinese non-small cell lung cancer patients. Support Care Cancer. 2015;23:635-41.

\section{Submit your next manuscript to BioMed Central and we will help you at every step:}

- We accept pre-submission inquiries

- Our selector tool helps you to find the most relevant journal

- We provide round the clock customer support

- Convenient online submission

- Thorough peer review

- Inclusion in PubMed and all major indexing services

- Maximum visibility for your research

Submit your manuscript at www.biomedcentral.com/submit
Ciomed Central 